\title{
Colloidal CdSe Nanocrystals Synthesized in Noncoordinating Solvents with the Addition of a Secondary Ligand: Exceptional Growth Kinetics
}

Quanqin Dai, Dongmei Li, Haiyong Chen, Shihai Kan,* Hongdong Li, Shiyong Gao, Yuanyuan Hou, Bingbing Liu, and Guangtian Zou

National Laboratory of Superhard Materials, Jilin University, Changchun 130012, People's Republic of China

\section{Supporting Information}
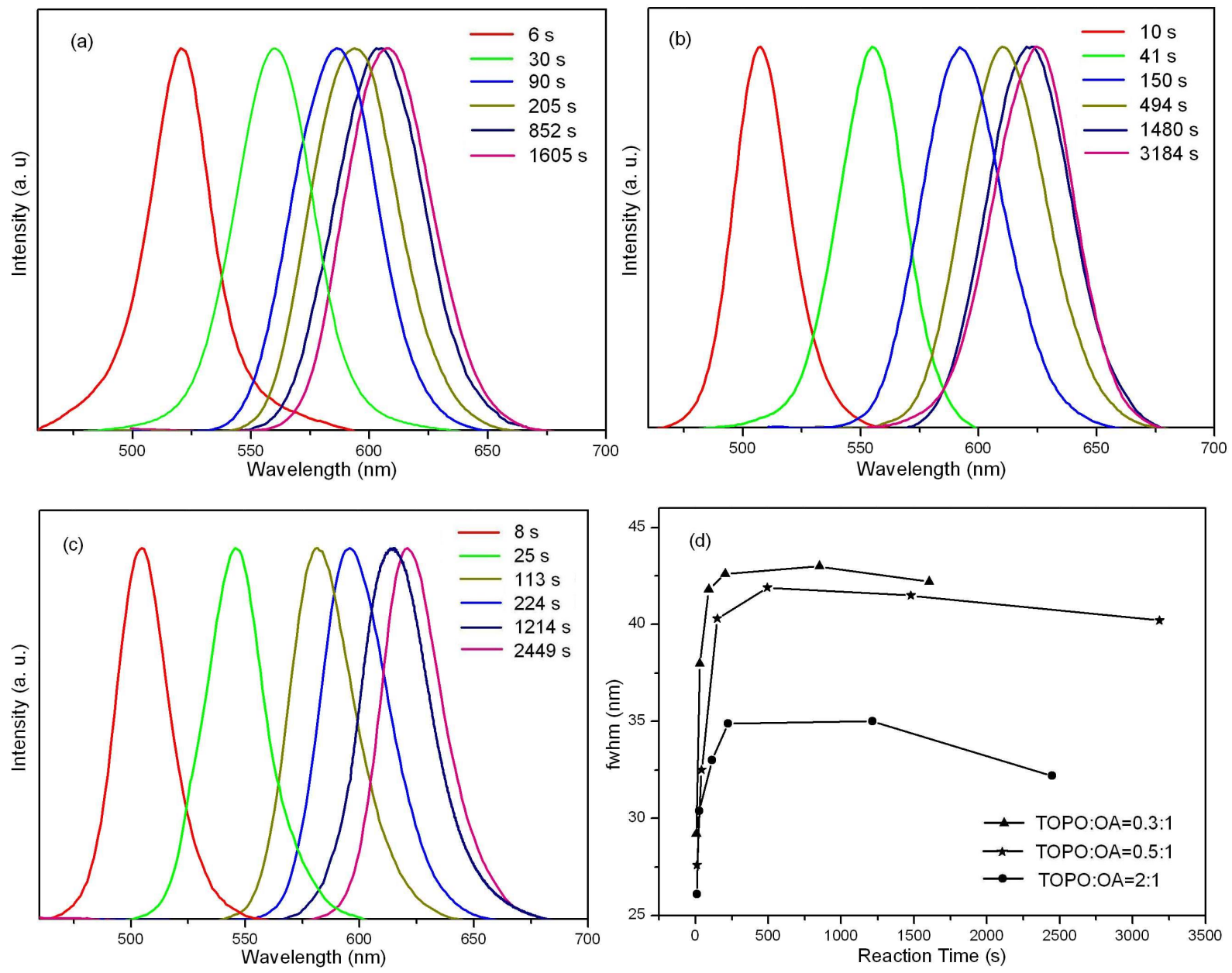
Figure S1. Temporal evolution of PL spectra of the CdSe nanocrystals grown in ODE with TOPO:OA molar ratios as of 0.3:1 (a), 0.5:1 (b), and 2:1 (c). (d) fwhm-indicated size distribution variation of the CdSe nanocrystals synthesized in ODE with the addition of a secondary ligand TOPO.

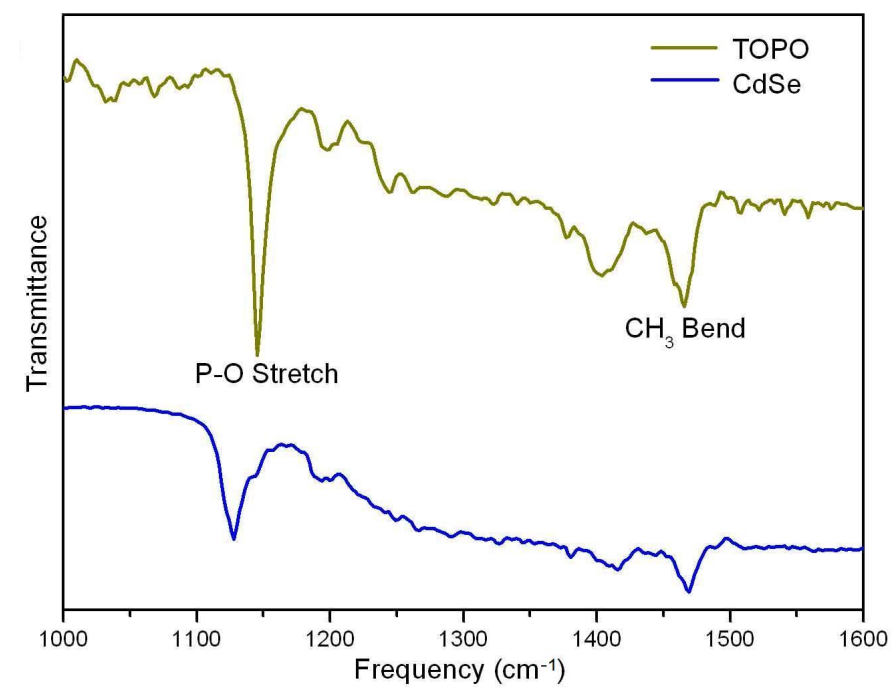

Figure S2. FTIR spectra of TOPO and the CdSe nanocrystals prepared with the existence of TOPO. The peaks of the nanocrystal spectra match those of the TOPO ones except for the P-O stretch, which shifts to lower frequency by about $20 \mathrm{~cm}^{-1}$ relatively to that of TOPO. Such shift is consistent with the previous report. ${ }^{1}$

\section{References}

(1) Bowen Katari, J. E.; Colvin, V. L.; Alivisatos, A. P. J. Phys. Chem. 1994, 98, 4109. 